\title{
Savior of Diabetes: Antioxidants
}

\author{
Zar Chi Thent and Azian Abd Latiff
}

Additional information is available at the end of the chapter

http://dx.doi.org/10.5772/intechopen.71748

\begin{abstract}
Introduction: The exposure of humans to antioxidants regulating the process and progress of diabetes mellitus (DM) is of major interest. Several phytoactive compounds such as flavonoids, lignans, prophenylphenols, etc. possess antioxidant property. Antioxidants exert free radical scavenging activity, improve the insulin sensitivity and pancreatic $\beta$ cell activity, stimulate insulin secretion, and reduce the carbohydrate absorption. Antioxidants also combat complications like diabetic wound healing by increasing the collagen deposition, improving the fibroblasts level, and decreasing the 11- $\beta$ hydroxydehydrogenase level. They revert the cardiovascular changes of DM by reducing the lipid profile level. Antioxidants also exert their regulatory effect on diabetic nephropathy and peripheral vascular diseases. Body-research methods: The terms "diabetes" or "diabetes mellitus" or type 1 diabetes mellitus" or "type 2 diabetes mellitus" or "hyperglycaemia" or "antioxidant" or "antioxidant" combined with "diabetic complication" were searched in following databases such as PubMed, Web of Science Scopus, and Google Scholar. Conclusion: Understanding the effects of antioxidants against DM is beneficial for disease progress assessment and development of prophylaxis regimens. Although several researches are carried out on antioxidants, current population has still less confidence on them. Hence, more detailed analysis and clinical studies investigating on the underlying mechanisms of antioxidants towards DM are mandatory.
\end{abstract}

Keywords: diabetes mellitus, antioxidant, prophylaxis, natural products, supplementation

\section{Introduction}

Diabetes mellitus (DM) is one of the most common noncommunicable diseases worldwide including Malaysia. Increasing prevalence of the disease both in developed and developing countries is of major concern. Based on the previous global statistical analysis, DM is 
expected to increase more than 400 million in the year 2030, which leads the disease to be the seventh leading cause of death worldwide [1]. DM is characterized as chronic hyperglycemia resulting due to insulin deficiency (type 1) or resistance (type 2). Both types of diabetes mellitus eventually develop various problems such as microvascular and macrovascular complications.

Chronic hyperglycemia is a multifaceted, progressive oxidative stress disorder results from imbalance between free radical formation and the antioxidant defense activity [2]. Several complications arise from diabetes mellitus (Figure 1). According to statistic, the high mortality rate among diabetic patients is due to the cardiovascular complications [3]. Numerous clinical and in vivo studies showed that diabetic state itself is responsible for developing

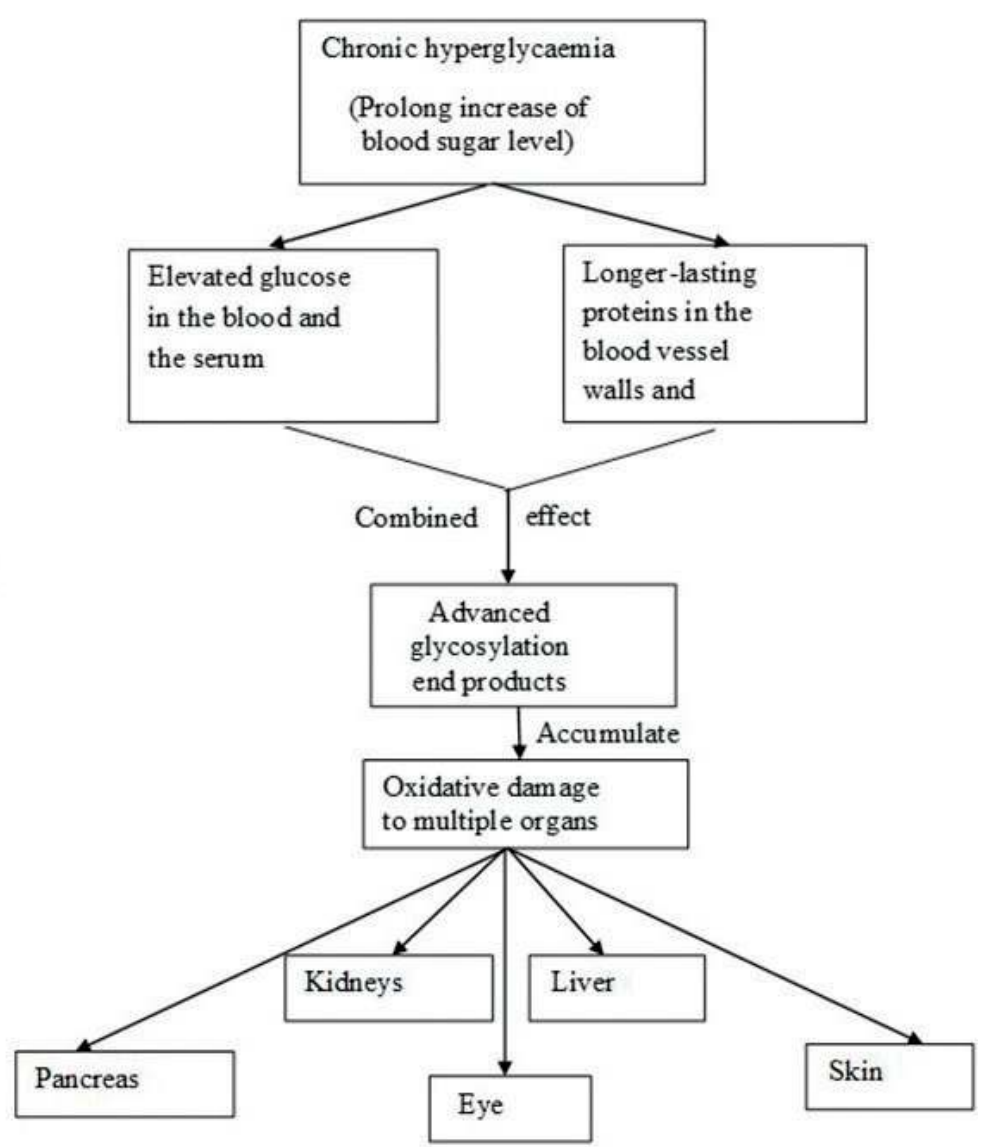

Figure 1. Flow chart shows the process of DM and its complications. 
cardiac dysfunction and atherosclerosis [4]. In addition, it also causes foot ulcers, impaired wound healing in association with peripheral neuropathy and autonomic dysfunction, and later results in limb amputation [5]. Amputation in diabetic patients is believed as global health burden in the current society [6].

In diabetic state, glucose combines with longer lasting proteins in blood-vessel walls and in the interstitial tissues. Following the event, the returning irreversible products called advanced glycosylation end-products (AGEs) are formed. DM is associated with increased formation of free radicals such as superoxide and reactive oxygen species (ROS) [7]. Therefore, the disturbance in oxidant and antioxidant activities, the increased formation of free radicals, and accumulation of AGEs lead to cause oxidative damage to multiple organs and systems of human body (Figure 2).

Over the years, the oxidative stress served as a common pathway for the pathogenesis of DM. Long-term DM leads to damages of the multiple organs like pancreas, liver, kidney, eyes, heart, and great vessels especially aorta and skin. Diabetic cardiomyopathy or cardiac dysfunction, microangiopathy, diabetic nephropathy, neuropathy, cataract, pancreatic $\beta$ cell destruction, nonalcoholic liver cirrhosis, poor wound healing, and erectile dysfunctions are the common complications that diabetic patients encountered to date [8]. Therefore, the stability and capacity of antioxidant status during the phase of DM seriously influence the outcome oxidative stress disorders like DM. Moreover, life-long treatment with high-cost modern drugs (biguanides, sulphonylurea, etc.) for DM is considered as a universal burden as well as believed to have several side effects [9]. Depending on the incidences of DM and its complications, it is important to explore the alternative source of antidiabetic supplement,

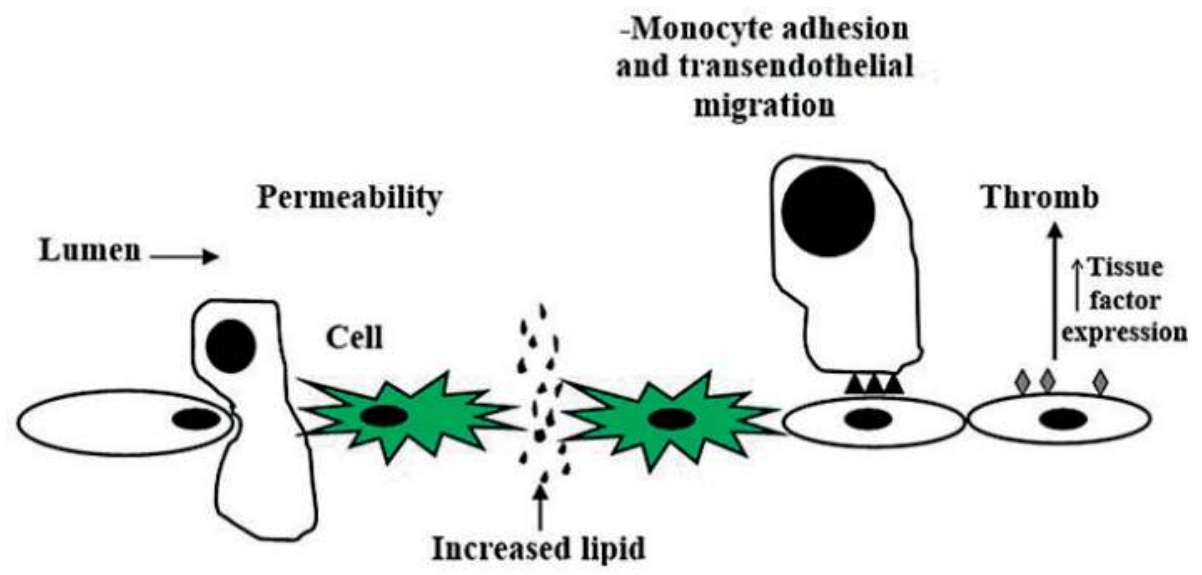

Subendoth

Figure 2. Diagram showing mechanism by which AGEs induce cardiovascular changes in DM [3]. 
which is enriched with antioxidant properties. We agreed the fact that there are plenty of natural products which have antioxidant property; however, only a few are highlighted in this chapter. Herein with, we present some common herbs and active compounds that are enriched with antioxidant activity.

\section{Antioxidants}

The decreased antioxidant level is found in the diabetic patient. In a previous study, the total antioxidant capacity in the plasma of type $1 \mathrm{DM}$ was shown to be $16 \%$ lower than that of normal subjects. Antioxidants counter the action of free radicals via several mechanisms such as degradation of free radicals, regulating the metals that stimulate the production free radicals, and scavenging the free radical. Lately, researchers found out the beneficial use of antioxidants from natural resources to replace synthetic ones.

Among the natural resources, data from laboratory studies showed that plants contain a large amount of antioxidant properties. Antioxidants occur in all parts of any higher plants (wood, bark, stems, pods, leaves, fruit, roots, flowers, pollen, and seeds). Plants with high levels of antioxidant have a significant role in improving oxidative stress disorders like DM. A number of findings showed the protective effect of the antioxidant ingredients against DM and achieved good results [10]. Hence, classical antioxidant could regulate the process and progress of DM and its complications. The good impact antioxidant activity toward DM and its associated complications has highly gained attention in the recent therapeutic society. Plenty of plants are rich in antioxidant property. To name it few, the plant or natural herbs such as Piper sarmentosum [11], Momordica charantia (bitter gourd) [12], and Piper betel [6], the common herbs used in improving the diabetic status enriched with antioxidant compounds. Noticeable active compounds like naringenin and quercetin are also important in managing hyperglycemic condition as well as regulating the oxidative stress-induced complication in DM. Vitamin $\mathrm{C}$ and $\mathrm{E}$ are well-known antioxidant agents for DM [13]. The antioxidant-enriched herbs not only increase the antioxidant level but also reduce the serum glucose level as well as improve the deteriorative changes in DM.

\subsection{Piper sarmentosum}

Piper sarmentosum (P.s), locally referred to as "daun kadok," is a member of the Piperaceae family which closely resembled the features of a betel leaf (Figure 3). Its leaves and roots are commonly used for experimental purposes [15]. P.s possesses high antioxidant compounds such as naringenin (75.7\%), hesperetin (91.7\%), taxifolin/dihydroquercetin (90.9\%), and quercetin $(98.1 \%)$ from its leaves [15].

During the past few years, our research group observed that type 1 diabetic rat treated with P.s groups showed a significant decrease $(\mathrm{P}<0.05)$ in fasting blood glucose level, urine glucose level [11], and blood pressure level [14] compared to untreated diabetic group in experimental Sprague-Dawley rats. The decrease in fasting blood and urine glucose level following P.s administration $(0.125 \mathrm{~g} / \mathrm{kg})$ is probably due to the underlying action of the antioxidant 


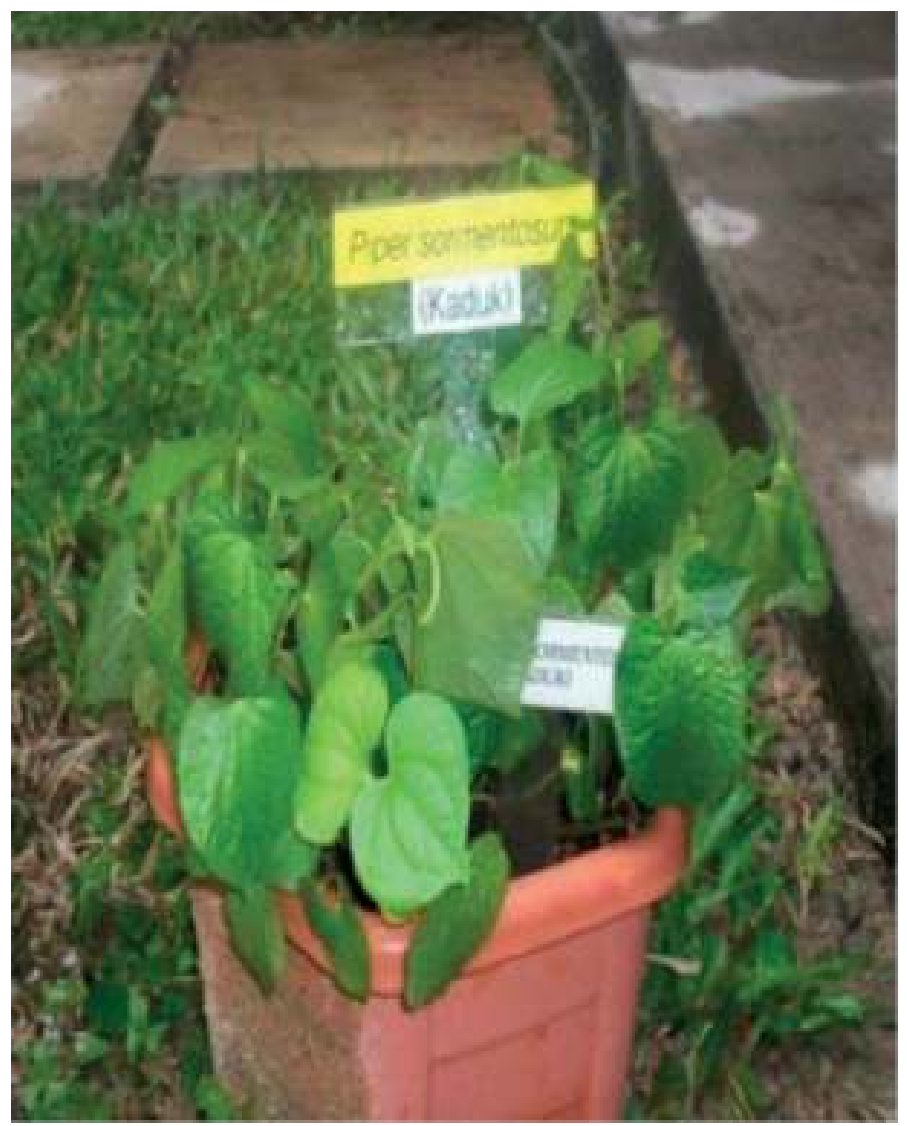

Figure 3. Photograph of Piper sarmentosum leaves [14].

compounds that are present in the leaves of P.s. Quercetin present in P.s has a positive role in reducing blood sugar levels, promoting the regeneration of the pancreatic islets and increasing insulin release with high superoxide scavenging activity [16].

Moreover, antioxidant compounds present in P.s prevent the morphological changes of diabetic cardiac and aortic tissues following 28 days of treatment (Figure 4 ). The antioxidant activity of quercetin controls the glucose uptake and increased levels of mitochondrial reactive oxygen species (ROS) linked to hyperglycemia [16]. This would protect the excess collagen deposition in cardiac tissues by inhibiting the metabolic disturbances of DM. Another antioxidant compound, naringenin, also improves endothelial function that reduces the risk of developing coronary heart disease [15]. The authors suggested that P.s could modify the cellular deteriorations in hyperglycemic condition. It was presumed that the mechanism by which P.s exerts its cardio-protective and vascular-protective effects might be due to the presence of antioxidant compounds [11]. 

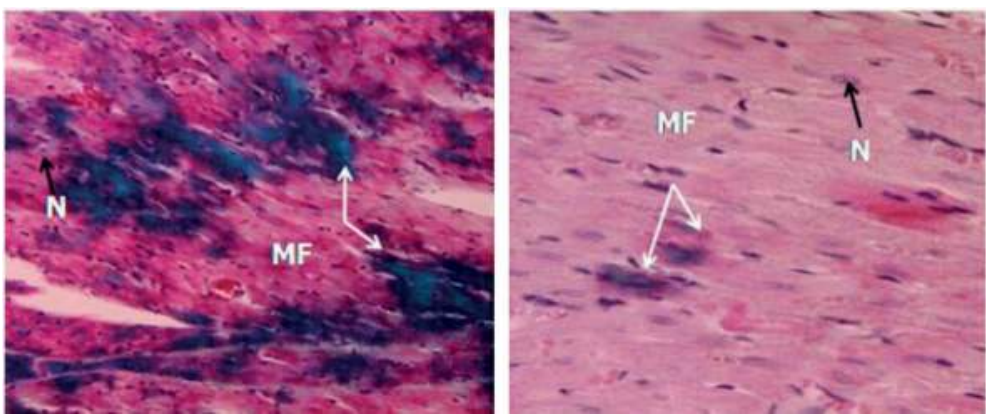

Figure 4. Photomicrograph showing the longitudinal section of cardiac tissues of untreated diabetic group (Left) diabetic group treated with $0.125 \mathrm{~g} / \mathrm{kg}$ Piper sarmentosum (Right). Note: N: nuclei of cardiomyocytes, MF: myofibers, white arrows: connective tissue under Masson's Trichome stain $(\mathrm{LM} \times 400)$ [17].

\subsection{Momordica charantia}

Momordica charantia (MC) belongs to the family of Cucurbitaceae (Figure 5) and is commonly known as bitter gourd or bitter melon [18]. The immature fruits of MC are a good source of Vitamin C and A. Several active compounds such as momorcharins, momordin, charantin, and goyasaponins are found in MC extract [19]. These are reported to present in all parts of the plant.

The leaf and fruit extract of MC are enriched with carbohydrate and protein. The supplementation with MC extract is a source of energy and nutrients for the body metabolic activities [20]. MC stimulates the number of pancreatic beta cells and promotes the insulin secretion.

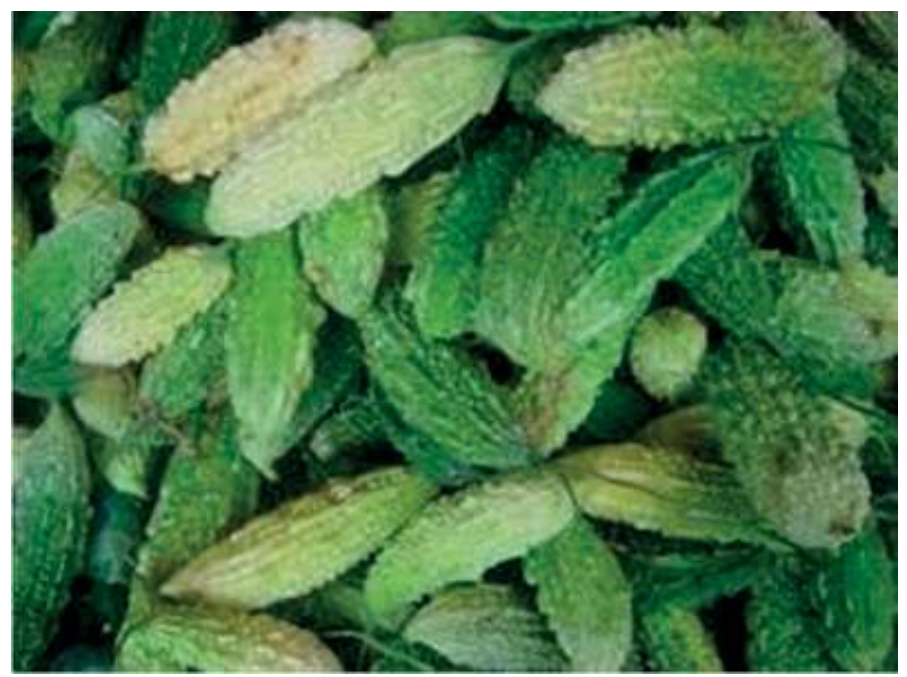

Figure 5. Fruits of Momordica charantia. 
Charantins from MC increase the glucose transporter (GLUT4), thus increases glucose utilization in the liver and muscle [18]. Increasing body weight that was observed in MC-treated experimental rats was due to the increase in glucose metabolism as compared to DM group [21]. Moreover, it was found out that the active compound present in MC fruit extract, leptin, lowered the blood pressure in diabetes. In the rural African communities, leptin is used in the management and control of DM-associated hypertension. Leptin reduces blood pressure by increasing the anti-oxidant and nitric oxide efficiency [22]. Our previous findings also showed significant decrease in systolic, diastolic, and mean blood pressure in experimental diabetic rats treated with $\mathrm{MC}$ [23].

In the diabetic state, there is a decrease in the activities of superoxide dismutase (SOD), glutathione, and catalase due to the increase production of ROS. Supplementation with $\mathrm{MC}$ extract restores anti-oxidant levels of SOD, glutathione, and catalase by regulating the deleterious effects of free radicals in diabetic state due to the presence of charantosides [24]. Moreover, supplementation with MC fruit extract also reverted the histological deteriorations of aortic tissue in diabetic rats (Figure 6). Apart from this, antioxidant compounds, luteolin, in the MC extract is observed to improve diabetic cardiac tissue morphology in experimental animals [12]. Previous study showed that MC fruit extract exerts positive effect on plasma MDA level in alloxan-induced diabetic. MC regulates membrane lipid peroxidation and reduces the thickness of diabetic aortic tissue with the presence of antioxidant effect [23].

\subsection{Piper betel}

Betel vine or scientifically known as Piper betel (PB), which belongs to Piperaceae family, is commonly found in South East Asia. PB is commonly found in South East Asian
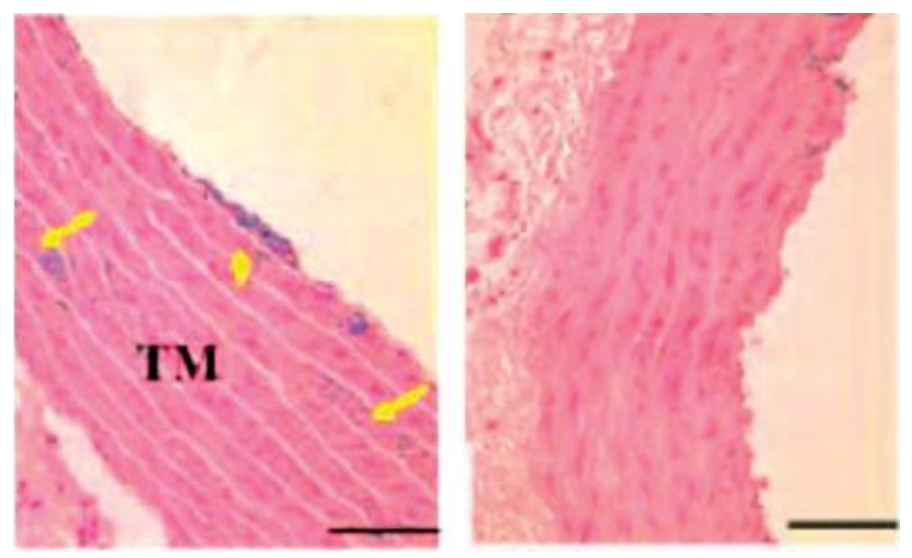

Figure 6. Photomicrograph showing transverse sections of the thoracic aorta under Alcian blue staining. Untreated diabetic group (Left) diabetic group treated with Momordica charantia fruit extract (Right). Note: TM = Tunica Media, $\mathrm{LM} \times 200)$ [23]. 
countries [25]. It was observed that $\mathrm{PB}$ is enriched with antioxidant activity by investigating on DPPH scavenging assay $[25,26]$. Researchers found the potent antioxidant property of PB suspension $(75 \mathrm{mg} / \mathrm{kg}$ ) in streptozotocin-induced diabetic animals. Eventually, it was believed that the antioxidant property of $\mathrm{PB}$ leaves plays a protective role in diabetes [27]. The topical application of PB $50 \mathrm{mg} / \mathrm{kg}$ for 10 days enhanced wound healing in diabetic rats [28].

Delayed wound healing is one of the critical complications in diabetes mellitus. Increased production of ROS and imbalance between oxidant and antioxidant enzymes contribute to impair healing process. In hyperglycemic condition, there is decreased superoxide dismutase (SOD) and increased malondialdehyde (MDA), a marker of lipid peroxidation and $11 \beta$ hydroxysteriod dehydrogenase-1 (11 $\beta$ HSD-1) enzyme, involving in the interconversion of cortisone and cortisol [29]. Increasing level of 11 $\beta$ HSD-1 enzyme has a negative influence on the fibroblasts proliferation. Less-responsive fibroblasts to growth factors in DM results in poor-wound tensile strength and decreased-wound closure rate [30]. Following 5 days of topical PB application to the diabetic wounds showed the fast wound closure rate (Figure 7) [7] as well as reduced $11 \beta$ HSD-1 enzyme expression in wound tissue (Figure 8) [8]. This might be due to the presence of potent active compounds in PB extract, which act as a free radical scavenger increase the anti-oxidant activity [32].

\subsection{Common active antioxidant compounds for diabetes}

Each compound contains its own efficacy. Naringenin (4',5,7-trihydroxyflavanone) is a highly potent natural antioxidant with high superoxide scavenging activity (Figure 9). Quercetin, an example of flavonoids, is also reported to improve the endothelial dysfunction by enhancing nitric oxide (NO) synthesis in human umbilical vein endothelial cells [16]. Tannins, the active potent anti-oxidant compounds, could increase the secretion of insulin and reduce hyperglycemia in experimental DM rats [34]. The mechanism of enhancing insulin secretion was clearly explained that the compounds contain an enzyme: a benzoic acid related molecules inhibited insulinase. This enzyme enhances the effect of insulin by inhibiting insulin degradation [34].
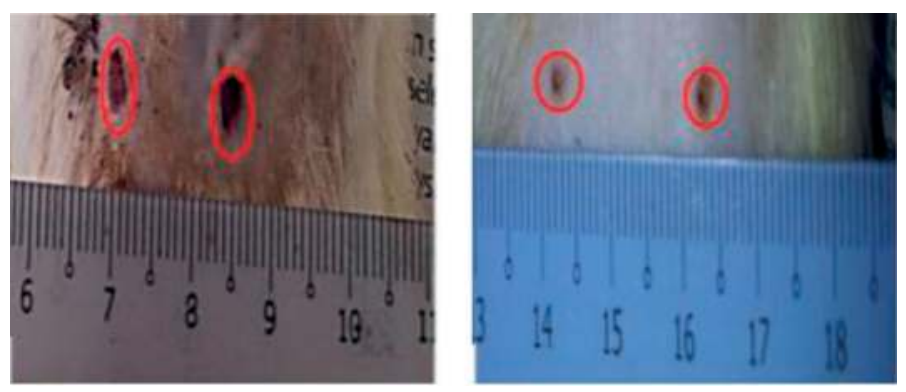

Figure 7. Increase in wound healing following topical piper betel application for 5 days. Untreated diabetic group (Left) diabetic group treated with PB extract (Right). Red circle = wounded area [31]. 

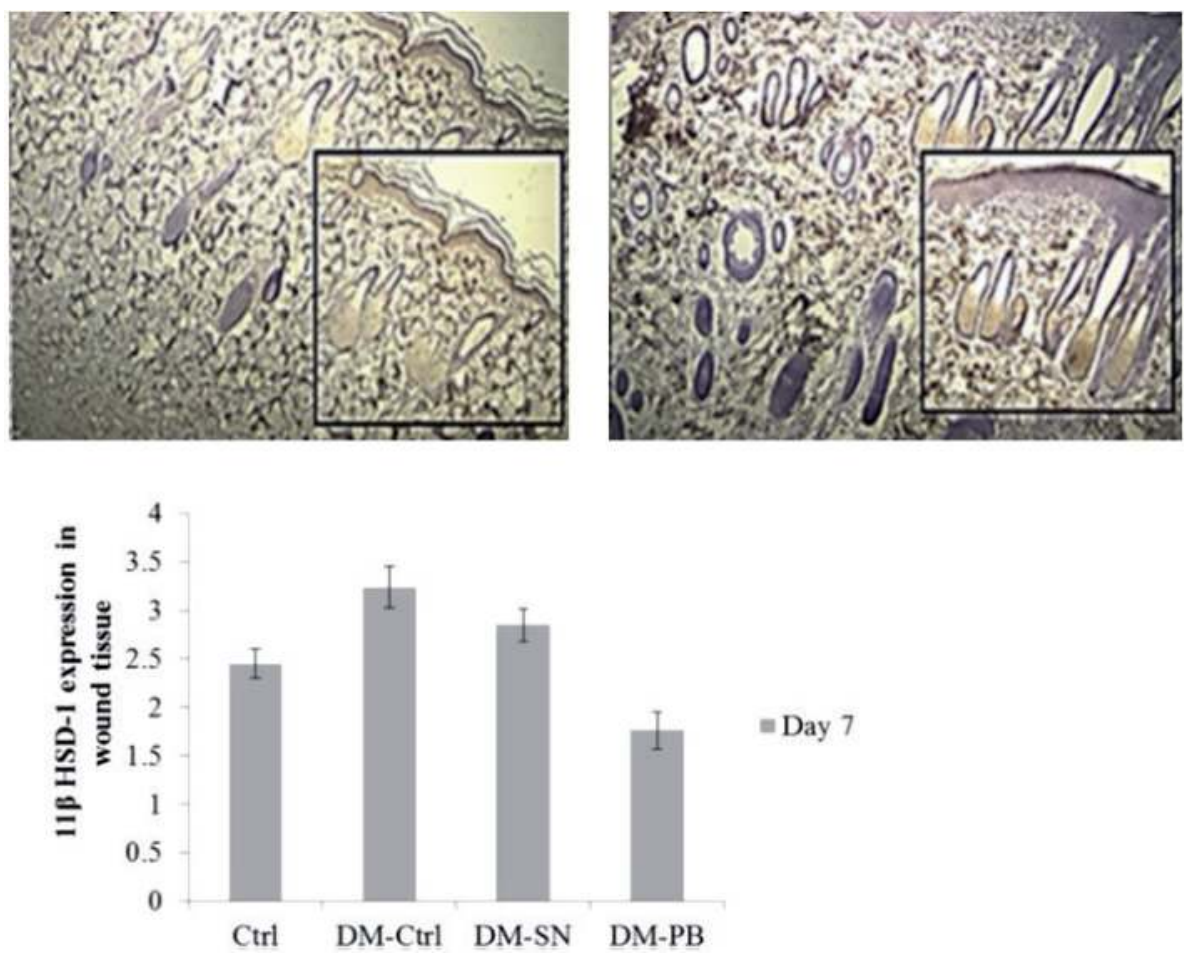

Figure 8. Expression of 11bHSD-1 by immunohistochemistry in wounded skin of diabetic rat model. Untreated diabetic group (Left) diabetic group treated with PB extract (Right). Decreased in expression was observed in the epidermal layer of the wounded skin of diabetic PB group compared to DM-Control (DM-Ctrl) and diabetic silver nitrate treated (DM-SN) groups [6].

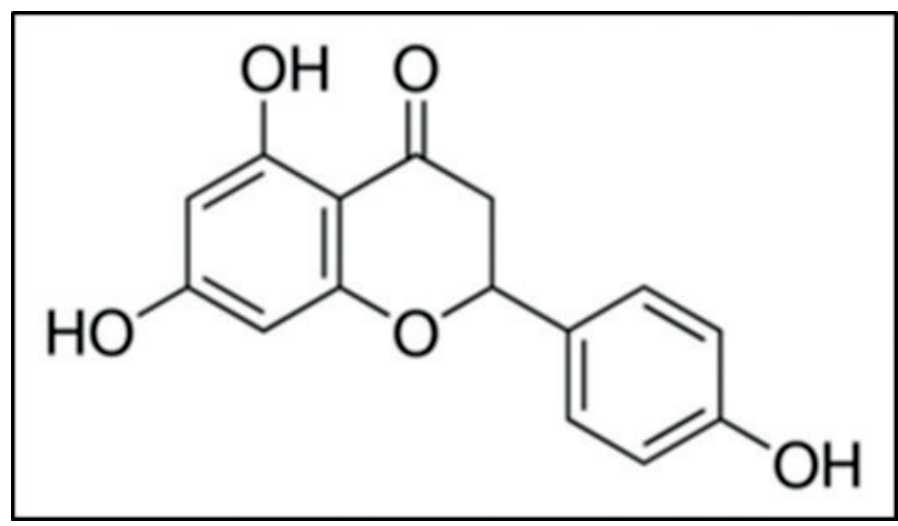

Figure 9. Chemical structure of Naringenin [33]. 


\section{Conclusion}

In conclusion, supplementation with antioxidant has been underestimated in modern society. However, studies have shown the positive findings of antioxidants against diabetes mellitus such as reducing the blood glucose level, regulating the disease complications such as cardiovascular complications, peripheral vascular disease like poor wound healing effect. Although natural sources of antioxidants are easily available worldwide, little attention is paid on its therapeutic usage. Detailed knowledge on the beneficial effects of antioxidants on oxidative stress disorder like diabetes mellitus is required. Understanding the underlying mechanism of antioxidant will be beneficial for disease progress assessment and development of prophylaxis regimens. It is believed that in future, supplements with antioxidant might serve as a savior of diabetes for general population.

\section{Author details}

Zar Chi Thent* and Azian Abd Latiff

*Address all correspondence to: zarrchii@gmail.com

Faculty of Medicine, Universiti Teknologi MARA, Sungai Buloh Campus, Jalan Hospital, Selangor, Malaysia

\section{References}

[1] Amos AF, McCarty DJ, Zimmet P. The rising global burden of diabetes and its complications: Estimates and projections to the year 2010. Diabetic Medicine. 1997;14(Suppl 5):S1-85

[2] Michiels C, Raes M, Toussaint O, Remacle J. Importance of se-glutathione peroxidase, catalase, and $\mathrm{Cu} / \mathrm{Zn}-\mathrm{SOD}$ for cell survival against oxidative stress. Free Radical Biology and Medicine. 1994;173:235-248. DOI: 10.1016/0891-584994;90079-5

[3] Aronson D. Cross-linking of glycated collagen in the pathogenesis of arterial and myocardial stiffening of aging and diabetes. Journal of Hypertension. 2003;211:3-12. DOI: 10.1097/01.hjh.0000042892.24999.92

[4] Bilim O, Takeishi Y, Kitahara T, Arimoto T, Niizeki T, et al. Diacylglycerol kinase zeta inhibits myocardial atrophy and restores cardiac dysfunction in streptozotocin-induced diabetes mellitus. Cardiovascular Diabetology. 2008;7:2. DOI: 10.1186/1475-2840-7-2

[5] Alberti KGMM, Zimmet PZ. Definition, diagnosis and classification of diabetes mellitus and its complications. Part 1: Diagnosis and classification of diabetes mellitus. Provisional report of a WHO consultation. Diabetic Medicine. 1998;157:539-553

[6] Ghazali NA, Elmy A, Yuen LC, Sani NZ, Das S, Thent ZC. Piper betel leaves induces wound healing activity via proliferation of fibroblasts and reducing $11 \beta$ hydroxysteriod dehydrogenase-1 expression in diabetic rat. Journal of Ayurveda and Integrative Medicine. 2016;74:198-208. DOI: 10.1016/j.jaim.2016.08.008 
[7] Naziroglu M, Butterworth PJ. Protective effects of moderate exercise with dietary vitamin $\mathrm{C}$ and $\mathrm{E}$ on blood antioxidative defense mechanism in rats with streptozotocininduced diabetes. Canadian Journal of Applied Physiology $=$ Revue Canadienne de Physiologie Appliquee. 2005;302:172-185

[8] Mytas DZ, Kyriazis IA. Diabetic cardiomyopathy. Archives of Hellenic Medicine. 2008; 254:432-441

[9] Sasvári M, Nyakas C. Time dependent changes in oxidative metabolism during chronic diabetes in rats. Acta Biologica Szegediensis. 2003;47:153-158

[10] Chanwitheesuk A, Teerawutgulrag A, Rakariyatham N. Screening of antioxidant activity and antioxidant compounds of some edible plants of Thailand. Food Chemsitry. 2005;92:491-497

[11] Thent ZC, Seong Lin T, Das S, Zakaria Z. Effect of piper sarmentosum extract on the cardiovascular system of diabetic sprague-dawley rats: Electron microscopic study. Evidence-Based Complementary and Alternative Medicine. 2012:1-9

[12] Abas R, Othman F, Thent ZC. Protective effect of Momordica Charantia fruit extract on hyperglycaemia-induced cardiac fibrosis. Razif. 2014;429060:1-8

[13] Penckofer S, Schwertz D, Florczak K. Oxidative stress and cardiovascular disease in type 2 diabetes: The role of antioxidants and pro-oxidants. Journal Cardiovascular Nursing. 2002;162:68-85

[14] Thent ZC, Das S. Piper Sarmentosum maintains blood pressure and morphological integrity of liver in type 1 diabetic rats. La Clinica Terapeutica. 2015;41:24-28

[15] Subramaniam V, Adenan MI, Ahmad AR, Sahdan R. Natural antioxidants: Piper sarmentosum Kadok; and Morinda elliptica Mengkudu. Malaysian Journal of Nutrition. 2003;91:41-51

[16] Vessal M, Hemmati M, Vasei M. Antidiabetic effects of quercetin in streptozocininduced diabetic rats. Comparative Biochemistry and Physiology Part C: Toxicology \& Pharmacology. 2003;1353:357-364

[17] Zar CT, Teoh SL, Srijit D, Zakaria Z. Histological changes in the heart and the proximal aorta in experimental diabetic rats fed with piper sarmentsoum. African Journal of Traditional, Complementary, and Alternative Medicines. 2012;9:396-404

[18] Alam S, Asad M, Asdaq SM, Prasad B, Raman VS, et al. Pharmacological actions and potential uses of Momordica Charantia: A review. Journal of Ethnopharmacology. 2015; 881:123-132

[19] Ansari N, Houlihan M, Hussain L, Pieroni B. Antioxidant activity of five vegetables traditionally consumed by south-Asian migrants in Bradford, Yorkshire, UK. Phytotherapy Research. 2005;1910:907-911

[20] Bakare R, Magbagbeola O, Akinwande AI, Okunowo O. Nutritional and chemical evaluation of Momordica Charantia. Journal of Medicinal Plants Research. 2010;421:2189-2193

[21] Sathishsekar D, Subramanian S. Antioxidant properties of Momordica Charantia bitter gourd; seeds on Streptozotocin induced diabetic rats. Asia Pacific Journal of Clinical Nutrition. 2005;142:153-158 
[22] Ojewole JA1, Adewole SO, Olayiwola G. Hypoglycaemic and hypotensive effects of Momordica Charantia Linn (Cucurbitaceae) whole-plant aqueous extract in rats. Cardiovascular Journal of South Africa. 2006;17(5):227-232

[23] Abas R, Othman F, Thent ZC. Effect of momordica charantia fruit extract on vascular complication in type 1 diabetic rats. EXCLI Journal. 2015;14:179-189

[24] Choo C, Waisundara VY, Hoon LY. Bittergourd (Momordica Charantia) scavenges free radicals by enhancing the expression of superoxide dismutase in in vitro models of diabetes and cancer. CyTA-Journal of Food. 2014;12:378-382

[25] Pradhan D, Suri KA, Pradhan DK, et al. Golden heart of the nature: Piper Betel. Journal of Pharmacognosy and Phytochemistry. 2013;16:147-167

[26] Santhakumari P, Prakasam A, KV P. Modulation of oxidative stress parameters by treatment with Piper betel leaf in streptozotocin induced diabetic rats. Indian Journal of Pharmacology. 2003;35:373-378

[27] Arambewela LSR, Arawwawala LDAM, Ratnasooriya WD. Antidiabetic activities of aqueous and ethanolic extracts of Piper betel leaves in rats. Journal of Ethnopharmacology. 2005;1022:239-245

[28] Keat EC, Razak SS, Fadil NM, Yusof FM, Chah LH, et al. The effect of Piper betel extract on the wound healing process in experimentally induced diabetic rats. Clinica Terapeutica. 2010;1612:117-120

[29] Singla R, Ganguli A, Ghosh M, Sohal S. Evaluation of sanitizing efficacy of acetic acid on Piper Betle leaves and its effect on antioxidant properties. International Journal of Food Sciences and Nutrition. 2009;60(Suppl):297-307

[30] Itoi S, Terao M, Murota H, Katayama I. 11 $\beta$-Hydroxysteroid dehydrogenase 1 contributes to the pro-inflammatory response of keratinocytes. Biochemical and Biophysical Research Communications. 2013;4402:265-270. DOI: 10.1016/j.bbrc.2013.09.065

[31] Sani NZ, Ghazali AN, Elmy A, Yuen LC, Thent ZC. Changes in the Body Weight and Wound Contraction Rate Following Treatment with Piper betel Extract in Diabetic Wounds Changes in the Body Weight and Wound Contraction Rate Following Treatment with Piper betel Extract in Diabetic Wounds. ICMEB:17 ${ }^{\text {th }}$ International Conference on Medical and Biomedical Engineering. Paris: France; 2015. pp. 35-40

[32] Wang BJ, Guo YL, Guo HR, Chang HY. Piper betle L. inflorescence causes allergic contact dermatitis of the hands during betel quid assembly. Contact Dermatitis. 2008;586:368370. DOI: 10.1111/j.1600-0536.2007.01295.x

[33] Kühnau J. The flavonoids. A class of semi-essential food components: Their role in human nutrition. World Review of Nutrition and Dietetics. 1976;24:117-191

[34] Marles RJ, Farnsworth NR. Phytomedicine. Antidiabetic plants and their active constituents. 1995;22:137-189. DOI: 10.1016/S0944-711311;80059-0 